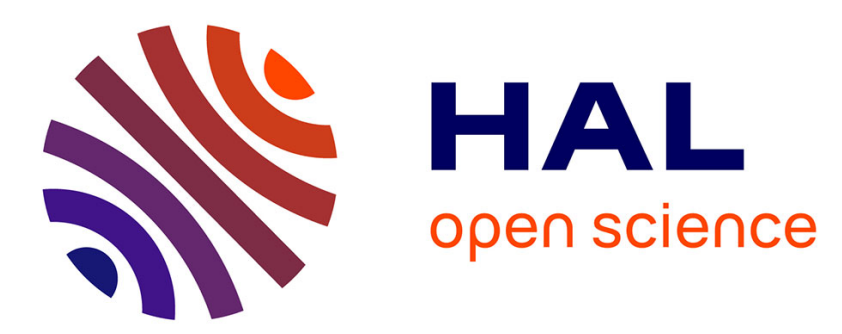

\title{
Characterization modeling and $\mathrm{H} \infty$ control of n-DOF Piezoelectric actuators: Application to a 3-DOF precise positioner.
}

\author{
Didace Habineza, Micky Rakotondrabe, Yann Le Gorrec
}

\section{- To cite this version:}

Didace Habineza, Micky Rakotondrabe, Yann Le Gorrec. Characterization modeling and H $\infty$ control of n-DOF Piezoelectric actuators: Application to a 3-DOF precise positioner.. Asian Journal of Control, 2016, 18 (5), pp.1-20. 10.1002/asjc.1224 . hal-01303480

\section{HAL Id: hal-01303480 \\ https://hal.science/hal-01303480}

Submitted on 18 Apr 2016

HAL is a multi-disciplinary open access archive for the deposit and dissemination of scientific research documents, whether they are published or not. The documents may come from teaching and research institutions in France or abroad, or from public or private research centers.
L'archive ouverte pluridisciplinaire HAL, est destinée au dépôt et à la diffusion de documents scientifiques de niveau recherche, publiés ou non, émanant des établissements d'enseignement et de recherche français ou étrangers, des laboratoires publics ou privés. 


\title{
Characterization, Modeling and $H_{\infty}$ Control of $n$-DOF Piezoelectric Actuators: Application to a 3-DOF Precise Positioner
}

\author{
Didace Habineza, Micky Rakotondrabe and Yann Le Gorrec
}

\begin{abstract}
This paper deals with the characterization, the modeling and the closedloop control of multivariable piezoelectric actuators, with an application to a 3-DOF piezoelectric tube scanner, widely used in precise positioning. These actuators are typified by hysteresis and creep nonlinearities, badly damped oscillation and strong couplings between their axis. First, during the modeling, we propose to decouple the system and to use a linear model where the couplings and the two nonlinearities are integrated through an external fictive disturbance. From the obtained monovariable systems, monovariable $H_{\infty}$ controllers are calculated by using specifications based on model approximation. The experimental tests demonstrate the efficiency of the method to reject simultaneously the couplings, hysteresis, creep and badly damped oscillations. Furthermore, the bandwidth of the closed-loop and the open-loop systems are compared and the results show that the proposed control technique allows to achieve a convenient closed-loop bandwidth and precision for all the axis of the precise positioner.
\end{abstract}

Key Words: Multi-axis (multi-degrees of freedom) piezoelectric actuators, hysteresis, creep, badly-damped oscillations, cross-couplings, characterization, modeling, $H_{\infty}$ control.

\section{INTRODUCTION}

Piezoelectric based actuators are very recognized as basis of precise positioning systems in nano/micro scale tasks. Comparatively to magnetostrictive, electrostatic and thermal actuators, piezoelectric actuators provide a higher positioning resolution, a large bandwidth and an ease of manufacturing and integration in positioning systems such as scanning and atomic force microscopy $[1,2,3]$ or in microrobotics [4]. In addition, beyond

\footnotetext{
Authors are with FEMTO-ST Institute, AS2M department, Université Bourgogne Franche-Comté/ Université de FrancheComté/CNRS/ENSMM,

24 rue Savary, 25000 Besançon, France.

This work has been supported by the national ANRJCJC C-MUMS-project (National young investigator project ANR-12-JS03007.01: Control of Multivariable Piezoelectric Microsystems with Minimization of Sensors). This work has also been supported by the Labex ACTION project (contract "ANR-11-LABX-01-01").
}

their actuation ability, piezoelectric actuators offer the possibility to be used also as sensors, by exploiting simultaneously piezoelectric direct and converse effects. Such possibility is called self-sensing [5, 6, 7, 8].

The most used piezoelectric actuators are piezocantilevers which are cantilevers with rectangular section and which are mainly used for microassembly and micromanipulation tasks [4, 9], piezostacks which are used for positioning and switching tasks [10,11], and piezo tube scanners (PTS) which are mainly used in scanning probe and atomic force microscopy [2].

Despite the advantages stated above, piezoelectric based actuators exhibit nonlinearities (hysteresis and creep), badly damped oscillations and high sensitivity to the thermal variation $[3,12,13]$. In addition, when dealing with multi-axis actuators, there are crosscouplings between the different axes that cause additional positioning inaccuracy $[14,15,17]$. 
To tackle these disadvantages, many research on their control have been conducted which include openloop and closed-loop techniques. Open-loop control of piezoelectric actuators is essentially based on model inversion, where the considered behavior (hysteresis, creep or oscillations) is first modelled and then the inverse model or its approximation is used as compensator, see for instance $[3,13,15,16,17,18,19$, $20,21,22,23]$ and references herein. This kind of control suffers from the lack of robustness face to model uncertainties and to external disturbances. Therefore, many works proposing feedback control, or open-loop combined with feedback techniques, have been raised, see for instance [3, 24, 25, 26, 27, 28, 29, 30, 31, 32, 33] and references herein. However, these works are only in concern with monovariable modeling and control.

Feedback control of multi-axis piezoelectric actuators has been investigated in [13, 34, 35, 36, 37, 38]. In these works, various feedback control techniques, including $H_{\infty}$, have been applied but the cross-couplings between the different axes of the actuator were not explicitely accounted for and were not identified for further consideration during the controller design. In [39] [40] [41], the cross-couplings were identified but the controllers were finally synthesized for frequency ranges outside the validity of these cross-couplings. Finally, an explicit and simultaneous consideration of the hysteresis, the creep, the dynamics and the crosscouplings in a generalized $n$-DOF (degrees of freedom), has not yet been treated.

The novelty of this paper is threefold: 1) simultaneous and explicit modeling of the hysteresis, of the creep, of the dynamics and of the cross-couplings in multi-DOF piezoelectric actuators, 2) systematic way to synthesize a $H_{\infty}$ controller for the yielded model, 3) and valuability of the proposed modeling and control techniques for any number of axes ( $n$-DOF). First, we propose a systematic approach to yield $n$ uncertain and perturbed linear monovariable systems from the initial $n$-DOF multivariable system. In the yielded model, the hysteresis is approximated by a linear gain followed by a direct multiplicative structured uncertainty and the creep and the cross-couplings are rassembled in a well defined and bounded fictive external disturbance. Afterwards, we propose $n$ decoupled $H_{\infty}$ controllers that explicitely account for the uncertainties, the disturbances and the specified performances. Thanks to the controllers, it is therefore possible to ensure the specifications on reference tracking in presence of the hysteresis, the creep, the badly damped vibrations and the cross-couplings. Experimental tests on a 3DOF piezotube actuator have been carried out and demonstrated the efficiency of the proposed modeling and control approach.

The paper is organized as follows: Section II reminds the modeling for monovariable piezoelectric actuators, and proposes the extension of this latter into a multivariable modeling valuable for $n$-DOF piezoelectric actuators. Section III is devoted to the $H_{\infty}$ control of multivariable piezoelectric actuators by using the model proposed in section II. In Section IV, the proposed modeling and $H_{\infty}$ control are applied to a 3-DOF positioner. Section $\mathrm{V}$ evaluates the performances of the proposed $H_{\infty}$ controller through various experimental tests. Finally, section VI concludes the paper.

\section{Modeling of $n$-DOF piezoelectric actuators}

\subsection{Remind of the monovariable modeling}

According to [42], the deflection $y$ of a cantilevered piezoelectric actuator with a nonlinear electromechanical part and subjected to an external force $F$ and a voltage $U$ (Fig. 1), can be expressed as:

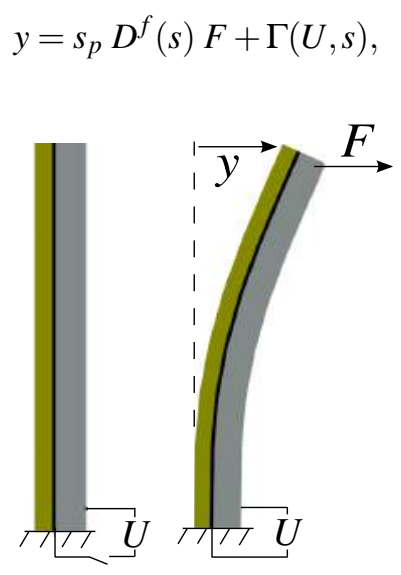

Figure 1: Deflection $y$ of a piezoelectric cantilever driven by a force $F$ and a voltage $U$.

where $s_{p}, D^{f}$ and $F$ represent the cantilever elastic constant, the mechanical dynamic part and the applied force, respectively. $s$ represents the Laplace variable and $\Gamma$ an operator which includes dynamic (or ratedependent) hysteresis and creep nonlinearities affecting the cantilever. In Eq. 1, the operator $\Gamma$ is a function of $U$ and $s$ since hysteresis depends upon the frequency or the rate and the history of the input $U$. In addition, the creep effect can be modelled by using dynamic 
transfers, which are functions of the Laplace variable $s[16,18]$.

The force $F$ is due to the contact between the actuator and the manipulated objects or other mechanical disturbances in the working environment. For the remainder of this paper, we consider $F$ to be negligible and keep the case where the actuator is driven only by the voltage $U$ :

$$
y=\Gamma(U, s) .
$$

Let us consider the modeling strategy developed in [30] where the nonlinear operator $\Gamma(U, s)$ is splitted into a dynamic or rate-dependent hysteresis operator $H(U, s)$ and a creep model $C^{r}(s)$, that is:

$$
y=\Gamma(U, s)=\underbrace{H(U, s)}_{\substack{\text { dynamic } \\ \text { hysteresis }}}+\underbrace{C^{r}(s) U}_{\text {creep }} .
$$

As classically admitted [3], the dynamic hysteresis $H(U, s)$ can be decomposed into a nonlinear static part $H_{s}(U)$ followed by a linear dynamic part $D(s)$. The hysteresis operator $H(U, s)$ can therefore be decomposed as in Eq. 4, where $H_{s}(U)$ represents the static (or rate-independent) hysteresis and $D(s)$ the normalized dynamic part $(D(s=0)=1)$.

$$
H(U, s)=H_{s}(U) D(s)
$$

The rate-independent hysteresis part $H_{S}(U)$ can be modelled by using classical nonlinear models such as Preisach [43], Prandtl-Ishlinskii [44] [45], BoucWen [46][47], etc. However, for sake of simplicity, [30] proposes quadrilateral modeling, which allows to determine a linear model for hysteresis, followed by a well modelled uncertainty. The proposed model has advantage of being simple and easy to identify, comparably to the aforementioned nonlinear models. This is particularly useful for multi-DOF systems where complex models lead to a difficult and long identification procedure.

Quadrilateral approximation consists in approximating a nonlinear curve by a piecewise affine function. Hence, a non saturated hysteresis curve is sufficiently modelled by a four sided polygon as in Fig. 2. This is generally the case for piezoelectric based actuators which exhibit non-saturated hysteresis within the input voltage $(U)$ operating range, differently to magnetostrictive or memory alloy actuators [48].

From this quadrilateral model, let us consider $K^{\max }$ and $K^{\text {min }}$ as the maximal and minimal slopes among the slopes of the four segments of the quadrilateral ABCD (slopes of straight lines $Y_{M}$ and $Y_{m}$, respectively). We

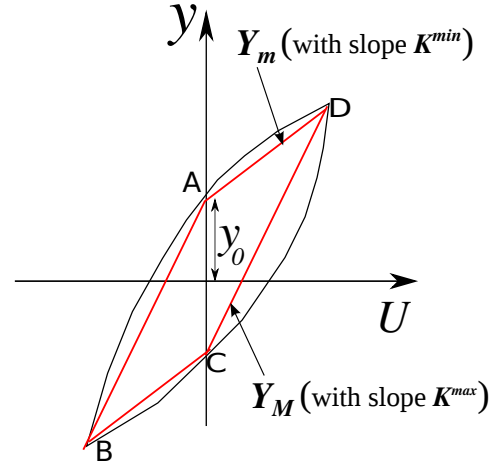

Figure 2: Quadrilateral modeling of hysteresis.

can now define three parameters: a static gain $(\alpha)$ which is the average (middle value) between $K^{\max }$ and $K^{\min }$, the uncertainty denoted $\delta$ on the static gain and a radius $r$ as in Eq. 5 .

$$
\left\{\begin{array}{l}
\alpha=\frac{K^{\max }+K^{\min }}{2} \\
r=\frac{K^{\max }-K^{\min }}{2} \\
-r \leq \delta \leq r
\end{array}\right.
$$

Hence, an uncertain affine model can be constructed as approximation of the rate-independent hysteresis:

$$
H_{s}(U)=(\alpha+\delta) U+y_{0},
$$

where $y_{0}$ is the (hysteresis) offset (see Fig. 2).

By replacing $H_{s}(U)$ in Eq. 4 with Eq. 6, we obtain the approximated dynamic model of the hysteresis:

$$
H(U, s)=(\alpha+\delta) D(s) U+y^{H},
$$

with $y^{H}=D(s) y_{0}$ which we will consider as a fictive disturbance further.

The identification of $K^{\max }$ and $K^{\min }$ permitting to yield $\alpha$ and $\delta$ is performed on a hysteresis curve captured at a frequency judiciously chosen, in order to isolate the rate-independent hysteresis from the phase lag and creep effects. In fact, at high frequency, the pure static hysteresis is affected by a phase shift between the input voltage and the actuator deflection. On the other hand, at a very low frequency, additional increase in deflection, due to the creep effect, will affect the rateindependent hysteresis [16].

The dynamic part $D(s)$ is identified from the step response of the actuator. In this case, systems identification techniques are used to derive the corresponding transfer function. Then, this derived transfer function is 
factorized in order to be normalized, i.e. in order to have $D(s=0)=1$.

Let us now combine the hysteresis approximation in Eq. 7 with the creep model in Eq. 3. We have:

$$
y=(\alpha+\delta) D(s) U+y^{H}+C^{r}(s) U,
$$

which can also be factorized as:

$$
y=\left(1+\frac{\delta}{\alpha}\right) \alpha D(s) U+y^{H}+C^{r}(s) U .
$$

This type of uncertainty can be expressed by using a direct multiplicative structure represented by a normalized uncertainty $\Delta$ weighted by a function $W$ as:

$$
y=(1+\Delta(s) W(s)) \alpha D(s) U+\underbrace{y^{H}+C^{r}(s) U}_{\text {disturbance } d},
$$

with

$$
\left\{\begin{array}{l}
\|\Delta\|_{\infty} \leq 1 \\
W=\frac{r}{\alpha}=\frac{K^{\max }-K^{\min }}{2 \alpha} .
\end{array}\right.
$$

The implementation of the model in Eq. 10 is represented by Fig. 3.

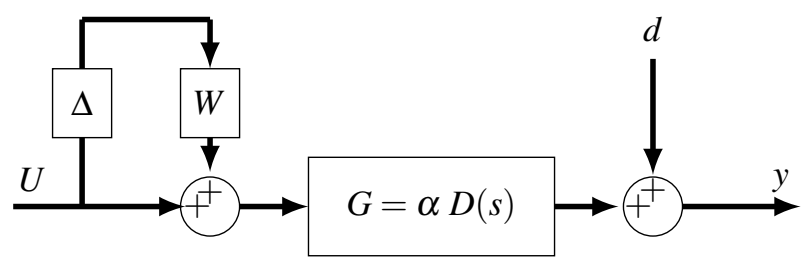

Figure 3: Implementation of the linear and dynamic model for the deflection $y$ of a piezoelectric actuator driven by a voltage $U . \Delta$ represents the normalized model uncertainty $\left(\|\Delta\|_{\infty} \leq 1\right)$ and $d$ the disturbance to the system, which includes the creep and hysteresis offset.

\subsection{Extension to multivariable modeling}

In this section, we propose to extend the monovariable modeling described above into a multivariable modeling, by considering a square multi-DOF piezoelectric system, with $n$ inputs and $n$ outputs as depicted in Fig. 4.

Let the monovariable representation in Eq. 10 be expressed as:

$$
\left\{\begin{array}{l}
y=k D(s) U+y^{H}+C^{r}(s) U \\
k=(1+\Delta(s) W(s)) \alpha .
\end{array}\right.
$$

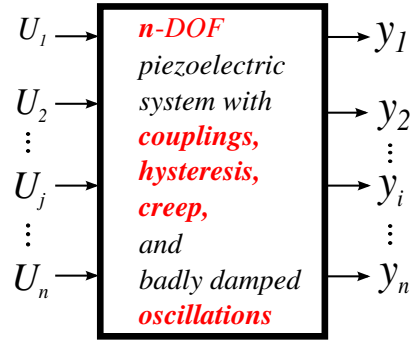

Figure 4: A multivariable system with hysteresis, creep, vibrations and cross-couplings.

From the first equation of Eq. 12, we propose the structure of Eq. 13 as a multivariable model, relating the input vector $U=\left(\begin{array}{llll}U_{1} & U_{2} & \cdots & U_{j} \\ \cdots & U_{n}\end{array}\right)^{T}$ and the output

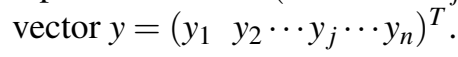

$$
\begin{array}{r}
\underbrace{\left(\begin{array}{c}
y_{1} \\
y_{2} \\
\vdots \\
y_{n}
\end{array}\right)}_{y}=\underbrace{\left(\begin{array}{cccc}
k_{11} & k_{12} & \cdots & k_{1 n} \\
k_{21} & k_{22} & \cdots & k_{2 n} \\
\vdots & \vdots & & \vdots \\
k_{n 1} & k_{n 2} & \cdots & k_{n n}
\end{array}\right)}_{K} \underbrace{\left(\begin{array}{cccc}
D_{11}(s) & D_{12}(s) & \cdots & D_{1 n}(s) \\
D_{21}(s) & D_{22}(s) & \cdots & D_{2 n}(s) \\
\vdots & \vdots & & \vdots \\
D_{n 1}(s) & D_{n 2}(s) & \cdots & D_{n n}(s)
\end{array}\right)}_{D(s)} \underbrace{\left(\begin{array}{c}
U_{1} \\
U_{2} \\
\vdots \\
U_{n}
\end{array}\right)}_{U} \\
+\underbrace{\left(\begin{array}{c}
y_{1}^{H} \\
y_{2}^{H} \\
\vdots \\
y_{n}^{H}
\end{array}\right)}_{y^{H}}+\underbrace{\left(\begin{array}{cccc}
C_{11}^{r}(s) & C_{12}^{r}(s) & \cdots & C_{1 n}^{r}(s) \\
C_{21}^{r}(s) & C_{22}^{r}(s) & \cdots & C_{2 n}^{r}(s) \\
\vdots & \vdots & & \vdots \\
C_{n 1}^{r}(s) & C_{n 2}^{r}(s) & \cdots & C_{n n}^{r}(s)
\end{array}\right)}_{C^{r}(s)} \underbrace{\left(\begin{array}{c}
U_{1} \\
U_{2} \\
\vdots \\
U_{n}
\end{array}\right)}_{U}
\end{array}
$$

where the operation $K \circ D(s)$ represents the Hadamard product between the two matrices: $(K \circ D(s))_{i, j}=$ $k_{i j} D_{i j}(s)$.

By developing Eq. 13, we can derive equation relating each single output $y_{i}$ to the input $U_{j}$ and the offset $y_{i}^{H}$ as follows:

$$
y_{i}=\underbrace{\sum_{j=1}^{n} k_{i j} D_{i j}(s) U_{j}}_{\text {hysteresis }}+\underbrace{y_{i}^{H}}_{\begin{array}{c}
\text { direct } \\
\text { hysteresis } \\
\text { offset }
\end{array}}+\underbrace{\sum_{j=1}^{n} C_{i j}^{r}(s) U_{j}}_{\text {creep }} .
$$

The subscripts $i$ and $j$ denote the considered output inside the vector $y$ and the considered input inside the vector $U$, respectively. When $i=j$ we have the direct transfers and when $i \neq j$, we have the cross-couplings.

By separating the direct and the cross-coupling in the hysteresis and creep terms, Eq. 14 becomes: 


$$
\begin{aligned}
y_{i}=\underbrace{k_{i i} D_{i i}(s) U_{i}}_{\text {direct hysteresis }} & +\underbrace{\sum_{\text {couplings hysteresis }}^{n} k_{i j} D_{i j}(s) U_{j}}_{\substack{j=1 \\
j \neq i}}+\underbrace{y_{i}^{H}}_{\begin{array}{c}
\text { direct } \\
\text { hysteresis } \\
\text { offset }
\end{array}} \\
& +\underbrace{C_{i i}^{r}(s) U_{i}}_{\text {direct creep }}+\underbrace{\sum_{\substack{j=1 \\
j \neq i}}^{n} C_{i j}^{r}(s) U_{j}}_{\text {couplings creep }} .
\end{aligned}
$$

Remind that the modeling strategy in this paper consists in considering the hysteresis offset, the creep and the cross-couplings as external fictive disturbances. Hence, Eq. 15 becomes:

$$
y_{i}=k_{i i} D_{i i}(s) U_{i}+\underbrace{y_{i}^{H}+d_{i i}^{C r}+\sum_{\substack{j=1 \\ j \neq i}}^{n} d_{i j}^{H}+\sum_{\substack{j=1 \\ j \neq i}}^{n} d_{i j}^{C r}}_{\text {disturbance } d_{i}},
$$

with $y_{i}^{H}$ the direct hysteresis offset, and $d_{i i}^{C r}, d_{i j}^{H}$, and $d_{i j}^{C r}$, standing for the worst cases of direct creep, coupling hysteresis and coupling creep, respectively. By worst case, we mean the maximal direct creep, the maximal couplings hysteresis and the maximal couplings creep obtained over the range of utilization, i.e. obtained with the maximal input voltage. Doing this will allow us to further calculate a controller which is robust even for the worst case.

Furthermore, accounting for the cross-coupling effect as a disturbance permits to study each axis $i$ $(1 \leq i \leq n)$ individually. In the sequel, the subscript $i i$ will be simply written as $i$.

Let us replace $k_{i}$ in Eq. 16 with the second equation of Eq. 12. The final model for axis $i$ whose the blockdiagram is shown is Fig. 5, is:

$$
\left\{\begin{array}{l}
y_{i}=\left(1+\Delta_{i}(s) W_{i}(s)\right) \alpha_{i} D_{i}(s) U_{i}+d_{i} \\
d_{i}=\left|y_{i}^{H}\right|+\left|d_{i}^{C r}\right|+\sum_{\substack{j=1 \\
j \neq i}}^{n}\left|d_{i j}^{H}\right|+\sum_{\substack{j=1 \\
j \neq i}}^{n}\left|d_{i j}^{C r}\right| .
\end{array}\right.
$$

Finally, from Eq. 17, the decoupled, linear and dynamic model, for a system with $n$ inputs and $n$ outputs, is derived (Eq. 18):

$$
\left(\begin{array}{c}
y_{1} \\
y_{2} \\
\vdots \\
y_{n}
\end{array}\right)=\left[\operatorname{diag}\left(\begin{array}{c}
\left(1+\Delta_{1}(s) W_{1}(s)\right) \alpha_{1} D_{1}(s) \\
\left(1+\Delta_{2}(s) W_{2}(s)\right) \alpha_{2} D_{2}(s) \\
\vdots \\
\left(1+\Delta_{n}(s) W_{n}(s)\right) \alpha_{n} D_{n}(s)
\end{array}\right)\right]\left(\begin{array}{c}
U_{1} \\
U_{2} \\
\vdots \\
U_{n}
\end{array}\right)+\left(\begin{array}{c}
d_{1} \\
d_{2} \\
\vdots \\
d_{n}
\end{array}\right)
$$

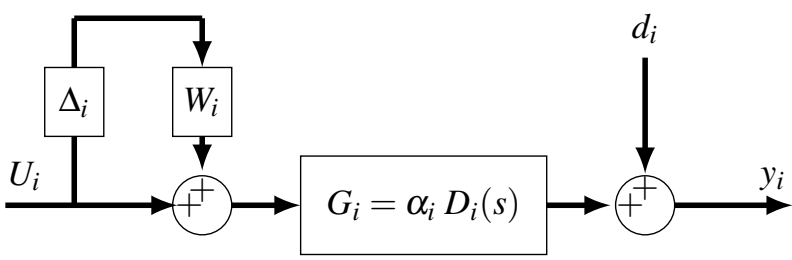

Figure 5: Implementation of the proposed model for axis $i(1 \leq i \leq n)$ : cross-couplings, creep and direct hysteresis offsets are included in the disturbance $d_{i} . \Delta_{i}$ represents the normalized model uncertainty $\left(\left\|\Delta_{i}\right\|_{\infty} \leq\right.$ $1)$.

\section{$3 \quad H_{\infty}$ control of $n$-DOF piezoelectric actuators}

This section is devoted to a systematic way of controller synthesize for the $n$-DOF piezoelectric actuator presented above and which was approximated by $n$ uncertain linear and perturbed monovariable system as described in Eq. 18. For that, we need first to characterize the hysteresis, the creep, the dynamics and the cross-couplings. This characterization is important to identify the model parameters and to characterize the uncertainties and the disturbance. Finally, after giving some specifications for the desired closed-loop performances, the $H_{\infty}$ synthesis problem is presented.

\subsection{Characterization of $n$-DOF piezoelectric actu- ators}

The steps followed to characterize the hysteresis, the creep, the dynamics and the cross-couplings for the $n$-DOF piezoelectric actuator are presented in this subsection.

\subsubsection{Hysteresis characterization}

In order to obtain experimental hysteresis curves for the direct transfers and for the cross-couplings, we apply a repetitive (sinusoidal or triangular) input voltage $U_{j}$ (starting by $j=1$ ) to the $n$-DOF system and leave all the remaining inputs (i.e. all $U_{k}$ with $k \neq j$ and $1 \leq k \leq n)$ null. A sinusoidal shaped input will yield a hysteresis corresponding to the frequency of the excitation signal whilst a triangular input will yield a hysteresis corresponding to different frequencies (the excitation frequency and the harmonics). In general, triangular input is employed when the final utilization of the actuator will employ such signal shape, like in atomic force microscopy application [3]. Then, we 
capture the actuator deflections $y_{i}$ due to this applied voltage $U_{j}$. The deflection $y_{i(i=j)}=\mathrm{f}\left(U_{j}\right)$ represents the direct hysteresis and all the remaining $y_{i(i \neq j)}=\mathrm{f}\left(U_{j}\right)$ are the cross-couplings. To obtain the hysteresis map for the whole system, we repeat the same procedure for all $U_{j}(2 \leq j \leq n)$.

\subsubsection{Creep characterization}

The creep nonlinearity is characterized by following the same steps than those of the hysteresis but, instead of utilizing a sine signal, step inputs are employed. The creep is observed as the slow drift on the step responses. For instance, in a piezoelectric cantilevered actuator with rectangular section, this drift can be observed over several tens of minutes [17]. The observed creep at the output $y_{i}$ due to a step input $U_{j}$ represents the direct creep when $i=j$, and represents the cross-couplings when $i \neq j$.

\subsubsection{Dynamics characterization}

Similarly to the creep, the dynamics are observed by applying step inputs. Then they are taken as the transient part in a very short period before the creep starts.

\subsection{Specifications and weighting functions}

From the system characterization above, let us specify the performances we desire for the closed-loop, in terms of tracking performances, command moderation and rejection of the disturbances.

For each monovariable system schematized in Fig. 5 and controlled by a controller $C_{i}(s)$, we systematically introduce three weighting functions called $W_{e}^{i}, W_{U}^{i}$ and $W_{d}^{i}$ related to the signals $e_{i}$ (error), $U_{i}$ (input signal) and $d_{i}$ (disturbance), respectively. We therefore obtain the augmented closed-loop in Fig. 6. In this, $y_{\text {iref }}$ is the reference input, $\tilde{e}_{i}$ and $\tilde{U}_{i}$ are the new outputs (also called weighted outputs) and $\tilde{d}_{i}$ is a new exogenous input. Applying the stability condition when having a direct multiplicative structure uncertainty (see for instance [49]), the uncertainty in Fig. 5 can be moved at the output signal $y_{i}$ in order to have only the weighting $W_{i}$ in the augmented closed-loop. We thus otbain the new augmented closed-loop schematized in Fig. 7. This latter scheme is used in next sections to define the standard $H_{\infty}$ problem and to compute the controller.

The desired specifications and corresponding weightings functions are defined as follows:

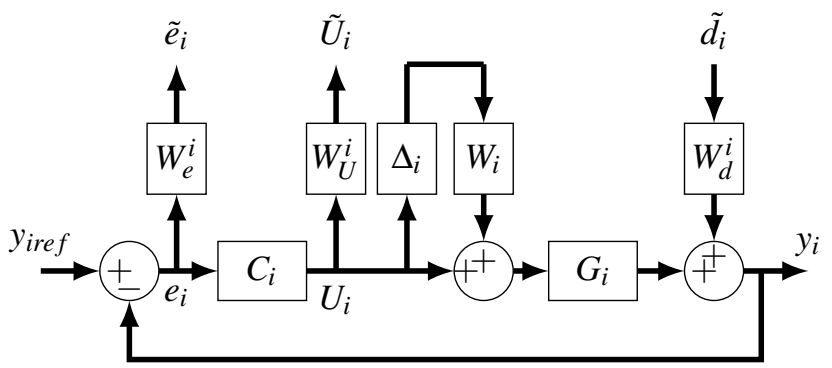

Figure 6: The closed-loop system augmented by the proposed weightings. $\Delta_{i}$ represents the normalized model uncertainty $\left(\left\|\Delta_{i}\right\|_{\infty} \leq 1\right)$.

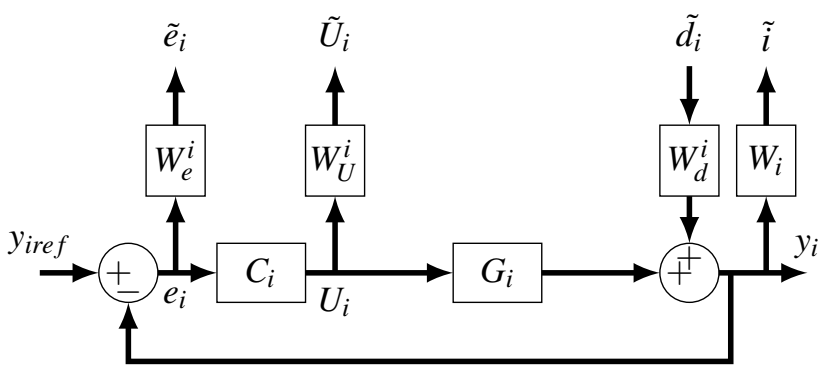

Figure 7: The representation from which the standard $H_{\infty}$ problem is defined (equivalent representation of the scheme of Fig. 6 in terms of stability condition).

- Tracking performances: in order to achieve good tracking performances for the closed-loop, we impose a maximal settling time $t_{i}^{r}$, a statical error less than $\varepsilon_{i}$ and finally, a zero overshoot $\left(v_{i}\right)$, for each axis $i$ of the actuator. A number of schemes to derive the gabarit $\frac{1}{W_{e}^{i}}$ (the inverse of the weighting $W_{e}^{i}$ ) has been proposed to account for these specifications [13] [50] [51]. In this paper, we use the following gabarit:

$$
\frac{1}{W_{e}^{i}}=\frac{\theta_{i} s+3 \varepsilon_{i} / t_{i}^{r}}{s+3 / t_{i}^{r}},
$$

whith $\theta_{i}=1+v_{i}$. For zero overshoot, $\theta_{i}=1$.

- Moderation of command signal $U_{j}$ : the objective of the command moderation is to limit the input control $U_{j}$ and thus, avoid over voltages which may saturate or destroy the actuator. Let us impose a maximal range of voltage $\left(U_{j}^{\max }\right)$ for the range of reference input $y_{\text {iref }}^{\max }$. Thus, the gabarit for the command moderation can be calculated as follows:

$$
\frac{1}{W_{U}^{i}}=\frac{U_{i}^{\max }}{y_{\text {iref }}^{\max }} .
$$


- Rejection of disturbances $d_{i}$ : the aim of this specification is to impose the performances on how to reject the disturbances $d_{i}$. For that, we set the settling time for disturbance rejection to be less than $t_{i}^{\text {rd }}$ for all the axis. In addition, we set the effect of the maximum disturbance $d_{i}$ to the corresponding output $y_{i}$ to be less than $\rho=$ $\left|y_{\text {iref }}-y_{i}\right|[\mu \mathrm{m}]$. The statical error under the effect of disturbance $\left(\varepsilon_{i}^{d}\right)$ is then given by:

$$
\varepsilon_{i}^{d}=\frac{\rho}{d_{i}^{\mathrm{wc}}},
$$

where $d_{i}^{\mathrm{wc}}$ represents the worst case of the disturbance $d_{i}$, calculated from the second equation of Eq. 17. From all that, we yield the gabarit related to the disturbance rejection:

$$
\frac{1}{W_{e}^{i} W_{d}^{i}}=\frac{\theta_{i} s+3 \varepsilon_{i}^{d} / t_{i}^{r d}}{s+3 / t_{i}^{r d}}
$$

- The uncertainty weightings: the uncertainty weightings $\left(W_{i}\right)$ are directly calculated from the second equation of Eq. 11.

\subsection{Standard $H_{\infty}$ problem and computation of the controller}

Having given the specifications and transformed them into gabarits (and therefore into weightings), we will use them in this subsection to synthesize the controllers $C_{i}(s)$ by employing the standard $H_{\infty}$ technique. The objective is to come into a matricial controller $C(s)$ of the structure represented by Eq. 23, able to satisfy the specifications imposed in the previous section.

$$
C(s)=\operatorname{diag}\left(C_{i}(s)\right)
$$

The coupling terms in the controller $C(s)$ are null due to the fact that the cross-couplings are already accounted in the disturbances $d_{i}$ for each axis model.

From the augmented closed-loop in Fig. 7, we yield the standard scheme for each axis as depicted in Fig. 8.

Thus, the standard $H_{\infty}$ problem consists in finding a controller $C_{i}(s)$ stabilizing the closed loop of Fig. 8 and satisfying the following constraint:

$$
\left\|F_{l}\left(P_{i}, C_{i}\right)\right\|_{\infty}<\gamma_{i},
$$

where $\gamma_{i}>0$ is the performances gain and $F_{l}\left(P_{i}, C_{i}\right)$ the lower linear fractional transformation transfer, which

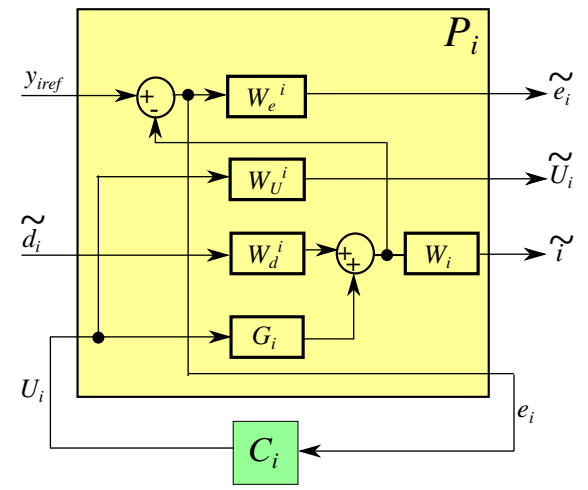

Figure 8: The standard scheme block diagram for each axis $i$.

links the exogenous inputs $\left(\begin{array}{ll}y_{i r e f} & \tilde{d}_{i}\end{array}\right)^{T}$ with the weighted outputs $\left(\begin{array}{lll}\tilde{e}_{i} & \tilde{U}_{i} & \tilde{i}\end{array}\right)^{T}$. It is defined by:

$$
\left(\begin{array}{c}
\tilde{e}_{i} \\
\tilde{U}_{i} \\
\tilde{e}_{i}
\end{array}\right)=\left[F_{l}\left(P_{i}, C_{i}\right)\right]\left(\begin{array}{c}
y_{\text {iref }} \\
\tilde{d}_{i}
\end{array}\right)
$$

i.e.

$$
F_{l}\left(P_{i}, C_{i}\right)=\left(\begin{array}{cc}
W_{e}^{i} S_{i} & -W_{e}^{i} S_{i} W_{d}^{i} \\
W_{U}^{i} C_{i} S_{i} & -W_{U}^{i} C_{i} S_{i} W_{d}^{i} \\
W_{i} G_{i} C_{i} S_{i} & W_{i} S_{i} W_{d}^{i}
\end{array}\right),
$$

where $S_{i}$ represents the sensitivity function, given by:

$$
S_{i}=\left(1+G_{i} C_{i}\right)^{-1}
$$

By combining Eq. 24 with Eq. 26, the problem becomes of finding the controller $C_{i}(s)$ such that the following condition is satisfied:

$$
\left(\begin{array}{cc}
\left\|W_{e}^{i} S_{i}\right\|_{\infty}<\gamma_{i} & \left\|-W_{e}^{i} S_{i} W_{d}^{i}\right\|_{\infty}<\gamma_{i} \\
\left\|W_{U}^{i} C_{i} S_{i}\right\|_{\infty}<\gamma_{i} & \left\|-W_{U}^{i} C_{i} S_{i} W_{d}^{i}\right\|_{\infty}<\gamma_{i} \\
\left\|W_{i} G_{i} C_{i} S_{i}\right\|_{\infty}<\gamma_{i} & \left\|W_{i} S_{i} W_{d}^{i}\right\|_{\infty}<\gamma_{i}
\end{array}\right),
$$

which is also satisfied if the following inequalities are respected:

$$
\left(\begin{array}{cc}
\left|S_{i}\right|<\left|\frac{1}{W_{e}^{i}}\right| \gamma_{i} & \left|S_{i}\right|<\left|\frac{1}{W_{e}^{i} W_{d}^{i}}\right| \gamma_{i} \\
\left|C_{i} S_{i}\right|<\left|\frac{1}{W_{U}^{i}}\right| \gamma_{i} & \left|C_{i} S_{i}\right|<\left|\frac{\frac{1}{W_{U}^{i} W_{d}^{i}} \mid \gamma_{i}}{\left|G_{i} C_{i} S_{i}\right|<\left|\frac{1}{W_{i}}\right| \gamma_{i}} \quad S_{i}\right|<\left|\frac{1}{\mid W_{i} W_{d}^{i}}\right| \gamma_{i}
\end{array}\right) .
$$

Each controller $C_{i}(s)$ of each problem in Eq. 24, $i=1 \rightarrow n$, can be afterwards calculated, in this case, by using the Doyle-Glover algorithm [52][53]. 


\section{Application to a 3-DOF nanoposi- tioner}

\subsection{Presentation of the experimental setup}

The 3-DOF positionner used is a piezotube scanner (PT230.94 from PIceramic company) which can provide deflections along $\mathrm{X}, \mathrm{Y}$ and $\mathrm{Z}$ axis as shown in Fig. 9. In fact, when a positive voltage $+U$ is applied to $+x$ electrode and a negative voltage $-U$ to -x electrode, the first sector expands while the second one contracts. This expansion and contraction result in a deflection of the overall piezotube along the $\mathrm{X}$ direction (Fig. 9-(c)). The same tube deflection is obtained along the $\mathrm{Y}$ direction if the voltages are applied to $+y$ and $-y$ electrodes (Fig. 9-(d)). The tube extension along $\mathrm{Z}$ axis is obtained by applying the same voltage $(+U$ or $-U)$ simultaneously on the four electrodes $+\mathrm{x},-\mathrm{x},+\mathrm{y}$ and $-\mathrm{y}$ (Fig. 9-(e)).

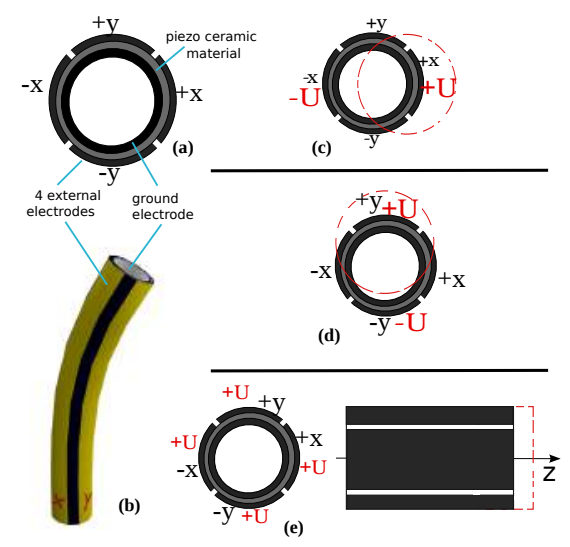

Figure 9: Piezoelectric tube structure and operation: (a) Top view, showing four external electrodes and an internal ground electrode, separated by piezoelectric material; (b) A perspective view of the piezoelectric tube; (c) deflection along $\mathrm{X}$ axis; (d) deflection along $\mathrm{Y}$ axis, and (e) elongation along $\mathrm{Z}$ axis.

We use a dSPACE board and a computer (with Matlab Simulink) to generate the control signals $U_{x}$, $U_{y}$ and $U_{z}$ and to further implement the controller. A high voltage amplifier $( \pm 200 \mathrm{~V})$ amplifies these driving signals before supplying the piezotube. The displacements $x, y$ and $z$ are measured with three inductive displacement sensors from IBS company (ECL202). These sensors have a bandwidth of $15 \mathrm{kHz}$, a resolution of $40 \mathrm{~nm}$ and a measurement range of $500 \mu \mathrm{m}$. Fig. 10 presents the experimental setup.

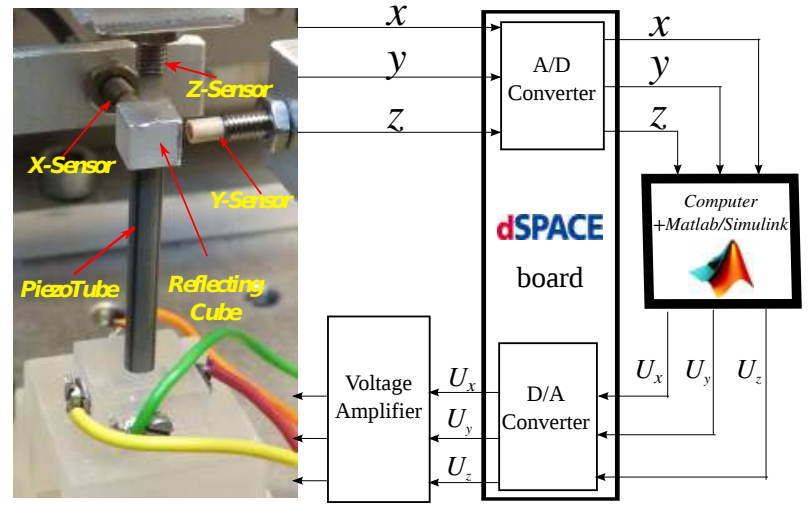

Figure 10: Experimental setup description.

\subsection{Characterization of the 3-DOF piezoelectric positioner}

\subsubsection{Hysteresis characterization}

Hysteresis has been characterized by using the procedure described in Section 3.1.

We apply first a sine input signal $U_{x}$ of $200 \mathrm{~V}$ and leave all other input signals $\left(U_{y}\right.$ and $\left.U_{z}\right)$ equal to zero. The frequency of the sine input signal was chosen to be $f=0.1 \mathrm{~Hz}$. As stated in Section 2.1, the frequency at which the rate-independent hysteresis is characterized has to be judiciously chosen, in order to isolate the rateindependent hysteresis from the phase lag and creep effects. From the characteristics of the actuator used, the frequency $f=0,1 \mathrm{~Hz}$ was found to be a good compromise. The application of the signal $U_{x}$ causes the bending of the tube along $X$ axis. However, we also observe bendings along $\mathrm{Y}$ and $\mathrm{Z}$ axis due to the cross-couplings presence. These cross-couplings can be caused by the minor misalignment of the electrodes of the actuator which results in a diagonal bending instead of purely axial bendings. Fig. 11-(a),(d) and (g), depict the displacement $x$ vs. $U_{x}, y$ vs. $U_{x}$ and $z$ vs. $U_{x}$, respectively. Afterwards, we repeat the previous step by using a sine input $U_{y}$ and by letting $U_{x}$ and $U_{z}$ be equal to zero. Fig. 11-(b),(i) and (h), depict $x$ vs. $U_{y}, y$ vs. $U_{y}$ and $z$ vs. $U_{y}$, respectively. Finally, we use a sine input $U_{z}$ and let $U_{x}$ and $U_{y}$ be equal to zero. Fig. 11-(c),(f) and (i) depict $x$ vs. $U_{z}, y$ vs. $U_{z}$ and $z$ vs. $U_{z}$, respectively.

The curves in Fig. 11-(a),(e) and (i) represent the direct hysteresis. The remaining curves represent the couplings hysteresis. 

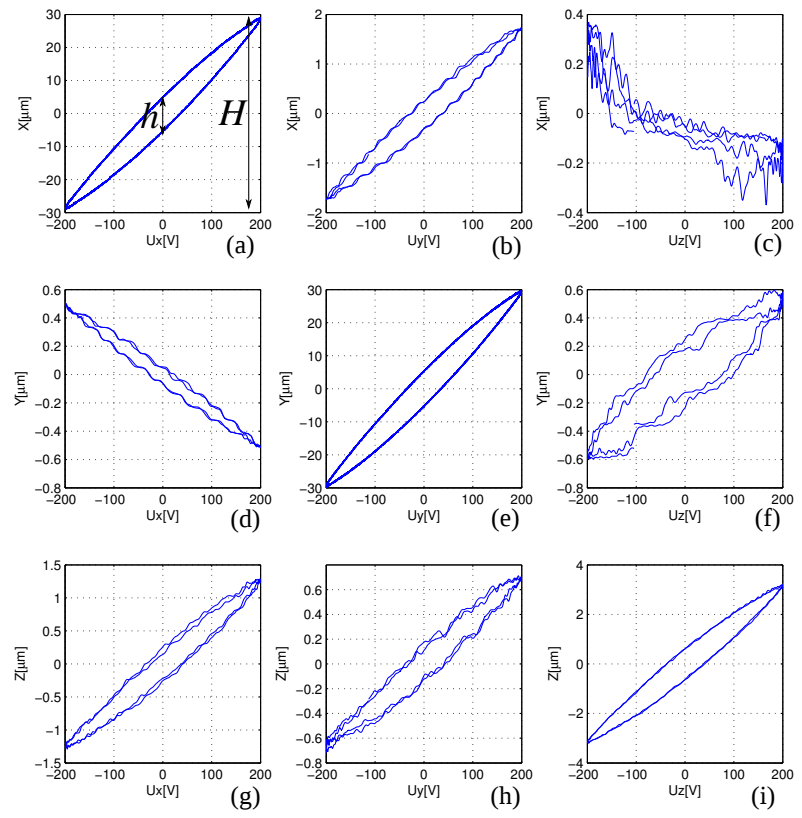

Figure 11: Hysteresis characterization with input sinusoidal signals of amplitude $200 \mathrm{~V}$ and of frequency $0.1 \mathrm{~Hz}$. Notations $h$ and $H$ in (a) are used later for computation of numerical values of hysteresis.

\subsubsection{Creep characterization}

The creep nonlinearity was characterized by applying a step inputs of amplitude equal to $200 \mathrm{~V}$. In this paper, the creep is observed over 600s. Fig. 12 depict the results where we observe direct creeps (Fig. 12-(a),(e) and (i)) and the couplings creeps (Fig.12-(b),(c),(d),(f),(g) and (h)). As we can see, the creep can have positive or negative evolution.

\subsubsection{Dynamics characterization}

Similarly to the creep, the dynamics are observed by applying step inputs of amplitude equal to $200 \mathrm{~V}$ and observing the step response during a short period of about $20 \mathrm{~ms}$, corresponding to the transient part. Fig. 13(a),(e) and (i) show the direct step responses while Fig. 13-(b),(c),(d),(f),(g) and (h), show the cross-couplings step responses. These figures demonstrate the lightly damped oscillations in the different responses.

\subsection{System modeling}

We follow the modeling procedure in Section 2.2, in order to propose a linear, dynamic and decoupled
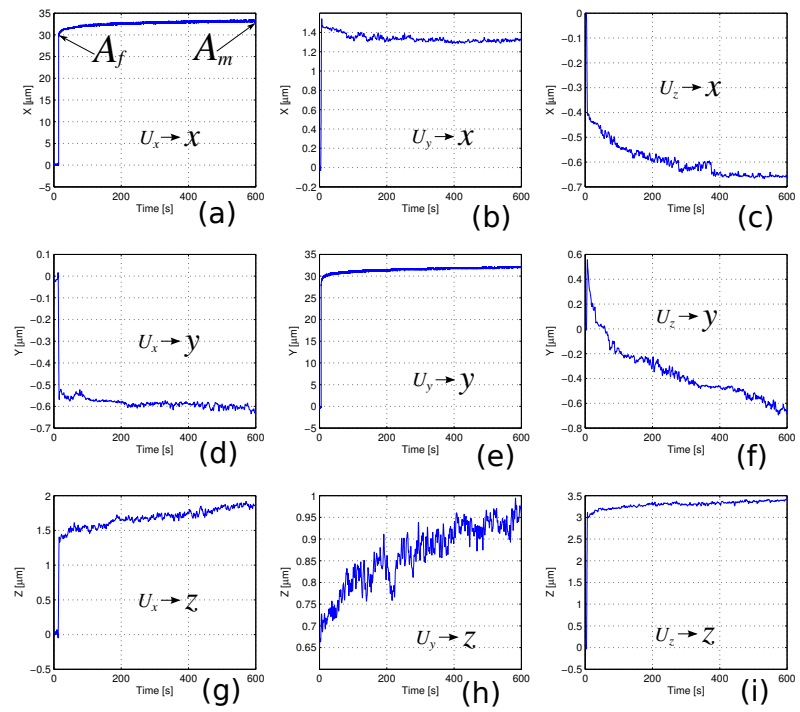

Figure 12: Creep characterization: step responses obtained with input steps of amplitude $200 \mathrm{~V}$ and observed over a period of $600 s$. Notations $A_{f}$ and $A_{m}$ in (a) are used later for computation of numerical values of creep.
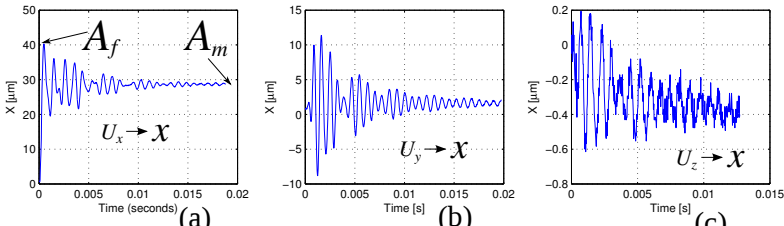

(b)
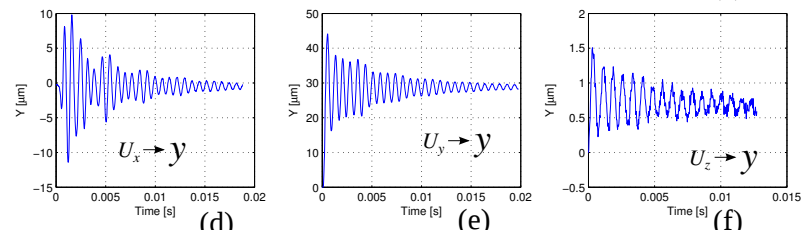

(d)
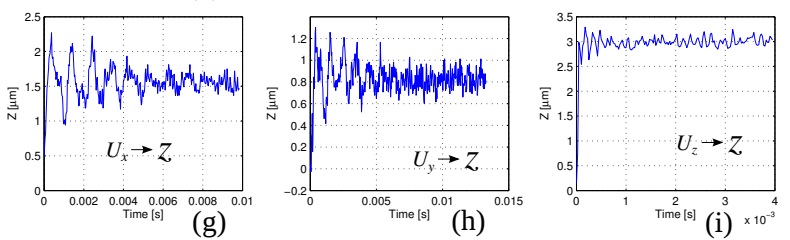

Figure 13: Dynamics characterization: transient responses obtained with step inputs of amplitude $200 \mathrm{~V}$ and observed during 20ms. Notations $A_{f}$ and $A_{m}$ in (a) are used later to numerically quantify the oscillations.

model for the 3-DOF piezotube, and which captures the hysteresis, creep, dynamics and cross-couplings. 


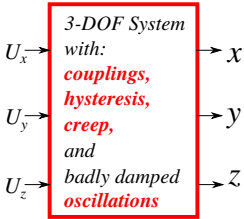

(a)

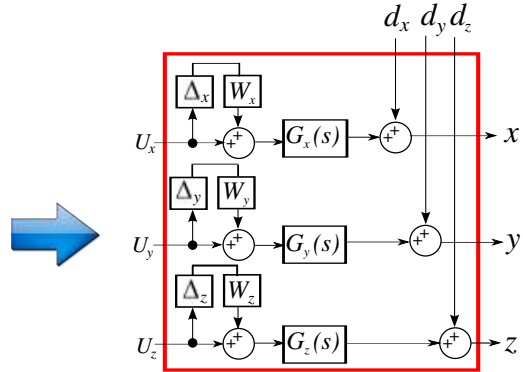

(b)
Figure 14: (a): the nonlinear, oscillating and coupled 3DOF system. (b): approximation into 3 monovariable systems with external fictive disturbance.

For that, the initial nonlinear, oscillating and coupled 3-DOF system pictured in Fig. 14-(a) is rewritten to be equivalent to the system in Fig. 14-(b), which contains 3 monovariable systems each one with uncertainty and external fictive disturbance. Derived from Eq. 18, the model corresponding to the 3-DOF piezotube is:

$$
\left(\begin{array}{l}
x \\
y \\
z
\end{array}\right)=\left[\operatorname{diag}\left(\begin{array}{c}
\left(1+\Delta_{x}(s) W_{x}(s)\right) \alpha_{x} D_{x}(s) \\
\left(1+\Delta_{y}(s) W_{y}(s)\right) \alpha_{y} D_{y}(s) \\
\left(1+\Delta_{z}(s) W_{z}(s)\right) \alpha_{z} D_{z}(s)
\end{array}\right)\right]\left(\begin{array}{l}
U_{x} \\
U_{y} \\
U_{z}
\end{array}\right)+\left(\begin{array}{l}
d_{x} \\
d_{y} \\
d_{z}
\end{array}\right),
$$

where $W_{i}, \alpha_{i}, D_{i}(s)$ and $d_{i}(i \in\{x, y, z\})$ are the parameters to be identified.

\subsection{Identification of the model parameters}

\subsubsection{Identification of $\alpha_{i}$}

Fig. 15 represents the quadrilateral modeling applied to each individual direct hysteresis (Fig. 11-(a),(e) and (i)), and the numerical values of slopes $K^{\max }$ and $K^{\min }$ for each axis.

By using the first equation of Eq. 5, the numerical values of $\alpha_{i}$ are obtained:

$$
\left\{\begin{array}{l}
\alpha_{x}=0.1400 \\
\alpha_{y}=0.1502 \\
\alpha_{z}=0.0150
\end{array}\right.
$$

\subsubsection{Identification of dynamics $D_{i}(s)$}

According to the model in Eq. 30, the dynamics to be identified are $D_{x}(s), D_{y}(s)$ and $D_{z}(s)$. By applying the ARMAX method to the individual experimental step responses of Fig. 13-(a),(e) and (i), we obtain transfer
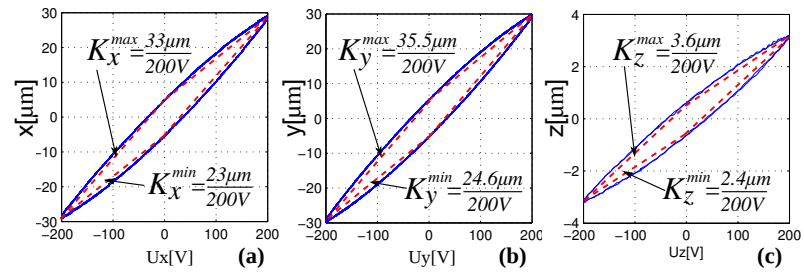

Figure 15: Experimental hysteresis curves (blue-solid line), quadrilateral modeling (red-dashed line) and numerical values of slopes $K^{\text {max }}$ and $K^{\text {min }}$, for each axis: (a), (b) and (c) represent hysteresis $x$ vs $U_{x}, y$ vs $U_{y}$ and $z$ vs $U_{z}$, respectively.

functions $G_{x}^{0}(s), G_{y}^{0}(s)$ and $G_{z}^{0}(s)$ (see Eq. 32), which are afterwards normalized in order to extract normalized dynamics $D_{x}(s), D_{y}(s)$ and $D_{z}(s)$ (see Eq. 33).

$$
\left\{\begin{array}{c}
G_{x}^{0}(s)=\frac{364.69(s+21.49)\left(s^{2}+6371 s+9.044 \times 10^{7}\right)}{(s+5784)(s+20.26)\left(s^{2}+728.4 s+4.043 \times 10^{7}\right)} \\
=0.1496 \frac{2438 s^{3}+1.558 \times 10^{7} s^{2}+2.208 \times 10^{11} s+4.738 \times 10^{12}}{s^{4}+6533 s^{3}+4.478 \times 10^{7} s^{2}+2.348 \times 10^{11} s+4.738 \times 10^{12}} \\
=0.1496 D_{x}(s) \\
G_{y}^{0}(s)=\frac{426.29(s+12.81)\left(s^{2}-3406 s+2.03 \times 10^{8}\right)}{(s+8832)(s+11.91)\left(s^{2}+374.1 s+6.893 \times 10^{7}\right)} \\
=0.1529 \frac{2787 s^{3}-9.459 \times 10^{6} s^{2}+5.658 \times 10^{11} s+7.251 \times 10^{12}}{s^{4}+9218 s^{3}+7.234 \times 10^{7} s^{2}+6.096 \times 10^{11} s+7.251 \times 10^{12}} \\
=0.1529 D_{y}(s) \\
G_{z}^{0}(s)=\frac{3.2654 \times 10^{12}}{\left(s+3.367 \times 10^{4}\right)\left(s^{2}+3.308 \times 10^{4} s+6.48 \times 10^{9}\right)} \\
=0.0150 \frac{2.182 \times 10^{14}}{s^{3}+6.675 \times 10^{4} s^{2}+7.594 \times 10^{9} s+2.182 \times 10^{14}} \\
=0.0150 D_{z}(s)
\end{array}\right.
$$

$$
\left\{\begin{array}{l}
D_{x}(s)=\frac{2438 s^{3}+1.558 \times 10^{7} s^{2}+2.208 \times 10^{11} s+4.738 \times 10^{12}}{s^{4}+65333^{3}+4.477 \times 10^{7} s^{2}+2.348 \times 10^{11}+4.738 \times 10^{12}} \\
D_{y}(s)=\frac{2787 s^{3}-9.459 \times 10^{6} s^{2}+5.658 \times 10^{11} s+7.251 \times 10^{12}}{s^{4}+9218 s^{3}+7.234 \times 10^{7} s^{2}+6.096 \times 10^{11} s+7.251 \times 10^{12}} \\
D_{z}(s)=\frac{2.182 \times 10^{14}}{s^{3}+6.675 \times 10^{4} s^{2}+7.594 \times 10^{9} s+2.182 \times 10^{14}}
\end{array}\right.
$$
1.

Notice that $D_{x}(s=0)=D_{y}(s=0)=D_{z}(s=0)=$

\subsubsection{Identification of disturbances $d_{i}$}

In this section, we identify numerically the maximum amount of external disturbances (worst case) affecting each axis.

Considering a 3-DOF system of inputs $U_{x}, U_{y}, U_{z}$ and outputs $x, y, z$, the disturbances expressed by the second equation of Eq. 17 are calculated as follows: 


$$
\left\{\begin{array}{l}
d_{x}=\left|y_{x}^{H}\right|+\left|d_{x x}^{C r}\right|+\left|d_{x y}^{H}\right|+\left|d_{x z}^{H}\right|+\left|d_{x y}^{C r}\right|+\left|d_{x z}^{C r}\right| \\
d_{y}=\left|y_{y}^{H}\right|+\left|d_{y y}^{C r}\right|+\left|d_{y x}^{H}\right|+\left|d_{y z}^{H}\right|+\left|d_{y x}^{C r}\right|+\left|d_{y z}^{C r}\right| \\
d_{z}=\left|y_{z}^{H}\right|+\left|d_{z z}^{C r}\right|+\left|d_{z x}^{H}\right|+\left|d_{z y}^{H}\right|+\left|d_{z x}^{C r}\right|+\left|d_{z y}^{C r}\right| .
\end{array}\right.
$$

From the characterization of the hysteresis and of the creep in figures Fig. 11 and Fig. 12, the numerical values of disturbances $d_{x}, d_{y}$ and $d_{z}$ are computed as:

$$
\left\{\begin{aligned}
d_{x} & =|5|+|5.2|+|1.5|+|-0.4|+|-0.2|+|-0.3| \\
& =12.6 \mu \mathrm{m} \\
d_{y} & =|5|+|4|+|-0.5|+|0.6|+|-0.1|+|-1.2| \\
& =11.4 \mu \mathrm{m} \\
d_{z} & =|0.6|+|0.5|+|1.5|+|0.8+| 0.3|+| 0.15|| \\
& =3.8 \mu \mathrm{m} .
\end{aligned}\right.
$$

\subsubsection{Identification of the uncertainty weightings $W_{i}$}

The Uncertainty weightings $W_{i}(i \in\{x, y, z\})$ are calculated from the second equation of Eq.11, with $K_{i}^{\max }$ and $K_{i}^{m i n}$ identified from the quadrilateral models in Fig. 15. We have:

$$
\left\{\begin{array}{l}
W_{x}=0.1786 \\
W_{y}=0.1814 \\
W_{z}=0.2000
\end{array}\right.
$$

\section{5 $H_{\infty}$ control of the 3-DOF piezoelectric posi- tioner}

\subsubsection{Specifications}

- Tracking performances: in order to improve the tracking performances of the actuator, we impose a settling time less than $10 \mathrm{~ms}$ for $\mathrm{X}$ and $\mathrm{Y}$ axis, and less than $0.5 m s$ for $\mathrm{Z}$ axis; a statical error less than $10 \%$; and finally a zero overshoot for all the three axes.

- Command moderation performances: the driving voltages of the actuator should be limited to avoid its saturation. For that, we impose a maximal input voltage amplitude $\left(U_{x}, U_{y}\right.$ and $\left.U_{z}\right)$ of $\pm 200 \mathrm{~V}$ for the maximal range of displacement. From the hysteresis identification in Fig. 11, this range is $\pm 30 \mu m$ for both $\mathrm{X}$ and $\mathrm{Y}$ axis, and $\pm 3 \mu \mathrm{m}$ for $\mathrm{Z}$ axis.
- Rejection of disturbances $d_{x}, d_{y}$ and $d_{z}$ : we set the settling time of disturbance rejection to be less than $5 m s$ for all the three axis. In addition, we set the effect of the maximal disturbance $d_{i}^{w c}$ for each axis, to be less than $1 \mu \mathrm{m}$.

Based on the schemes described in Section 3.2 and the gabarits in Eq. 29, these specifications have been transcribed into the following gabarits:

$$
\left\{\begin{array}{l}
\frac{1}{W_{e}^{x}}=\frac{s+60}{s+600} ; \frac{1}{W_{U}^{x}}=\frac{200}{30} ; \frac{1}{W_{e}^{x} W_{d}^{x}}=\frac{s+47.62}{s+600} \\
\frac{1}{W_{e}^{y}}=\frac{s+30}{s+300} ; \frac{1}{W_{U}^{y}}=\frac{200}{30} ; \frac{1}{W_{e}^{y} W_{d}^{y}}=\frac{s+5.263}{s+60} \\
\frac{1}{W_{e}^{z}}=\frac{s+60}{s+6000} ; \frac{1}{W_{U}^{z}}=\frac{200}{30} ; \frac{1}{W_{e}^{z} W_{d}^{z}}=\frac{s+1558}{s+6000}
\end{array}\right.
$$

\subsubsection{Calculation of the controller}

The objective is to calculate the controller $C(s)$ such that:

$$
C=\left(\begin{array}{ccc}
C_{x}(s) & 0 & 0 \\
0 & C_{y}(s) & 0 \\
0 & 0 & C_{z}(s)
\end{array}\right) .
$$

Hence, the $H_{\infty}$ problem of Eq. 29 has been solved for each axis $i$ (with $i=\{x, y, z\}$ ) and the three controllers $C_{x}(s), C_{y}(s)$ and $C_{z}(s)$ have been obtained:

$$
\left\{\begin{aligned}
& C_{x}(s)=\frac{39897(s+5784)(s+600)(s+54)(s+20)\left(s^{2}+728.4 s+4.0 \times 10^{7}\right)}{\left(s+1.7 \times 10^{4}\right)(s+600)(s+60)\left(s^{2}+35 s+692.9\right)\left(s^{2}+5933 s+6.3 \times 10^{7}\right)} \\
& \gamma_{x-o p t}=1.632359 \\
& C_{y}(s)=\frac{11358(s+8832)(s+300)(s+11.9)(s+8.4)\left(s^{2}+374.1 s+6.8 \times 10^{7}\right)}{\left(s+2.1 \times 10^{4}\right)(s+346.3)(s+30)(s+13)(s+1.0)\left(s^{2}+5393 s+1.5 \times 10^{8}\right)} \\
& \gamma_{y-o p t}=1.483102 \\
& C_{z}(s)=\frac{2.8 \times 10^{5}\left(s+2 \times 10^{5}\right)\left(s+3.3 \times 10^{4}\right)\left(s+6 \times 10^{3}\right)(s+1257)}{\left(s+2 \times 10^{5}\right)\left(s+3.9 \times 10^{4}\right)\left(s+6 \times 10^{3}\right)(s+1627)} \\
& \quad \times \frac{\left(s^{2}+3 \times 10^{4} s+6.4 \times 10^{9}\right)}{(s+60)\left(s^{2}+3 \times 10^{4} s+6.6 \times 10^{9}\right)} \\
& \gamma_{z-o p t}= 2.424572
\end{aligned}\right.
$$

where $\gamma_{i-o p t}(i \in\{x, y, z\})$ are the optimal values for the performances gains.

\section{Controller implementation and exper- imental tests}

\subsection{Simulation of the closed-loop frequency response}

Before implementing the controller, the bandwidth of the closed-loop has been evaluated. From the sensitivity function $S_{i}$ (Eq. 27), let $T_{i}$ (Eq. 40) be the closed-loop transfer function for axis $i(i=\{x, y, z\})$. 


$$
T_{i}=1-S_{i}=\left(1+G_{i} C_{i}\right)^{-1} G_{i} C_{i}
$$

Fig. 16 represents the bode diagrams of the identified transfer functions $G_{i}^{0}(s)$ and the closed-loop transfer functions $T_{i}(s)$. This figure shows that, with the calculated controller, the closed-loop achieves a convenient bandwidth relatively to the bandwidth of the open-loop system.
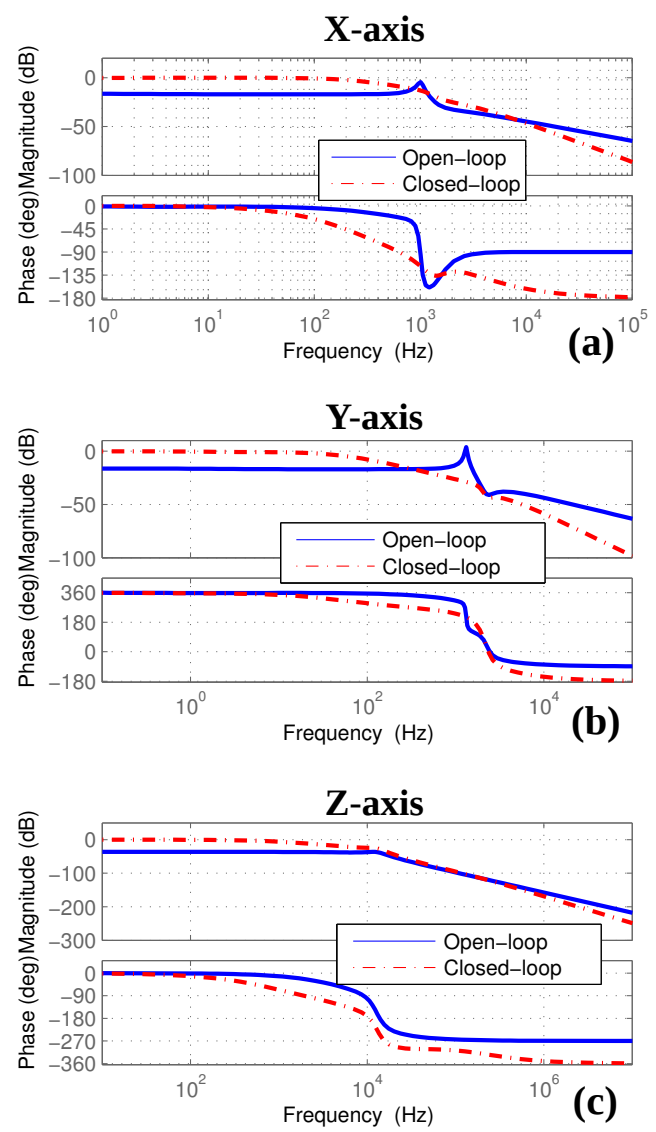

Figure 16: Open- and closed-loop frequency responses. (a), (b) and (c) for $\mathrm{X}, \mathrm{Y}$ and $\mathrm{Z}$ axis, respectively.

\subsection{Hysteresis, creep and dynamics for the closed- loop system}

After the simulation step, the calculated controller has been implemented (Fig. 17) by using SimulinkMATLAB software, and hysteresis, creep and dynamics for the closed-loop system have been characterized.

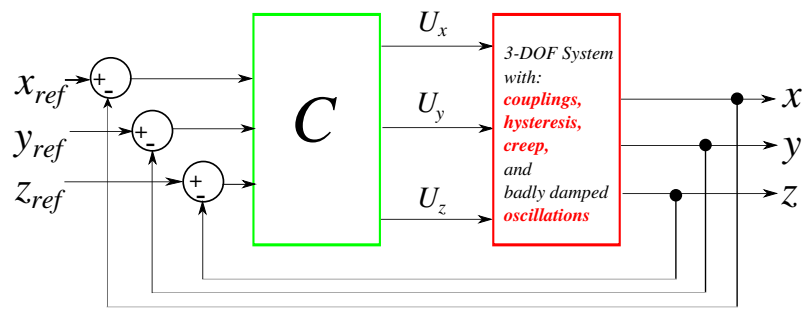

Figure 17: The closed-loop control of the 3-DOF positioner.

To characterize the hysteresis for the closed-loop system, input sine reference signals $x_{r e f}$ and then $y_{r e f}$ with an amplitude of $25 \mu \mathrm{m}$ and a frequency $f=$ $0.1 \mathrm{~Hz}$ have been applied successively. For $z_{\text {ref }}$, we used $3 \mu \mathrm{m}$ and $f=0.1 \mathrm{~Hz}$. The resulting outputs $x, y$ and $z$ are reported in Fig. 18. We notice the ability of the calculated controller to suppress hysteresis of direct transfers and the cross-couplings amplitudes.
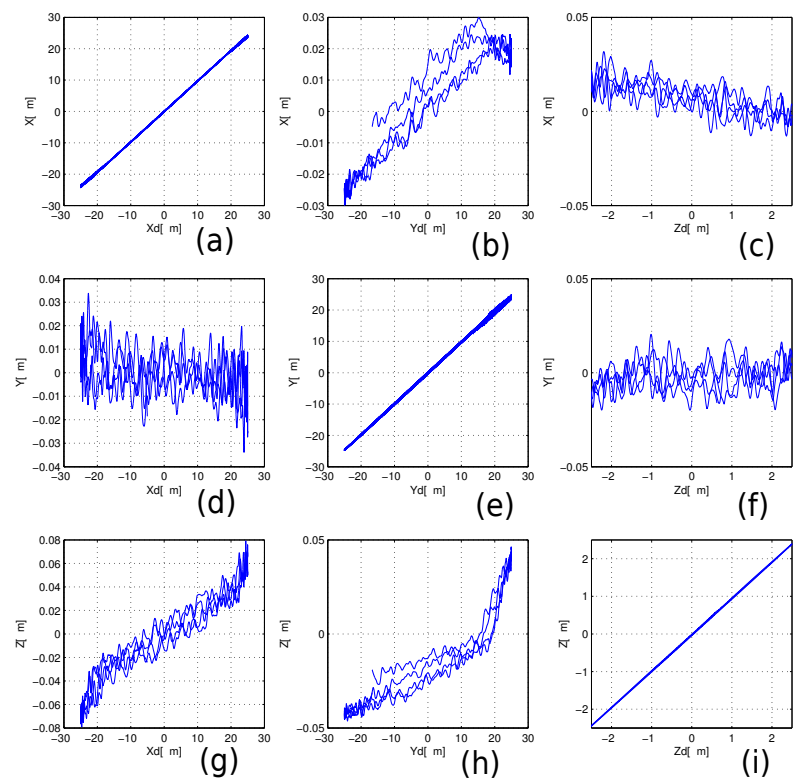

Figure 18: Verification of the hysteresis for the closedloop.

To observe the creep and dynamics for the closedloop system, step inputs of $25 \mu \mathrm{m}$ for $x_{r e f}$ and $y_{r e f}$, and of $3 \mu \mathrm{m}$ for $z_{\text {ref }}$ have been applied successively. While the creep are observed during 600s, the dynamics are observed during only $0.2 \mathrm{~ms}$.

The verification of the creep and dynamics for the closed-loop system is reported in Fig. 19 and Fig. 20, respectively. 
In Fig. 19, we notice the suppression of the creep and the stability of the closed-loop system during the observed period. The eight disturbances $d_{k}(k=1 \cdots 8)$ represent impulses applied intentionally (manually) to see the impact of disturbances on the controlled system. The manual impulses are applied to the mechanical support of the actuator. As we can see, disturbances are quickly rejected. Finally, dynamics in Fig. 20 show the convenient experimental settling times, the statical errors and the overshoot thanks to the calculated controller.
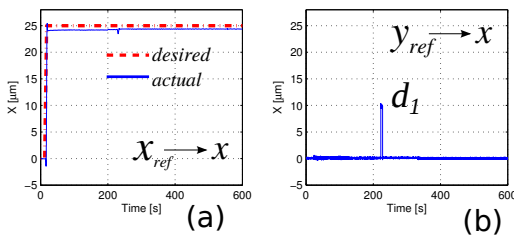

(b)
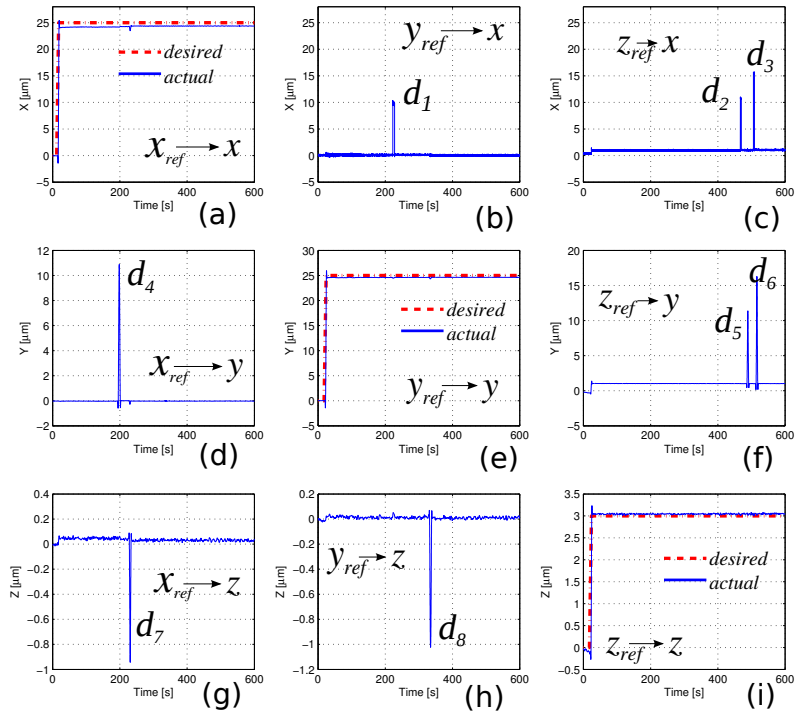

Figure 19: Verification of the creep for the closed-loop.

\subsection{Comparison between the open-loop and closed-loop systems: numerical values of hysteresis, creep, oscillations and cross-couplings}

To evaluate the impact of the calculated controller, various evaluation techniques, including graphical comparison of the system responses before and after the controller implementation, can be used.

In this subsection, we evaluate the effect of the calculated controller by comparing the numerical values of hysteresis, creep, oscillations and cross-couplings, before (open-loop) and after the implementation of the controller (closed-loop).

In this paper, to quantify numerically the hysteresis, we use the ratio $(h / H) \times 100 \%$, with $h$ and $H$ (see Fig. 11-(a)), the maximal residual deflection and the maximal (peak-to-peak) deflection, respectively.

To quantify the creep and oscillations, we use the expression $\left[\left(A_{m}-A_{f}\right) / A_{f}\right] \times 100 \%$. For the creep,

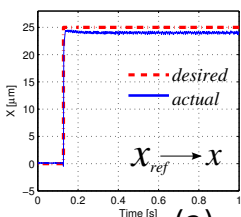

(a)

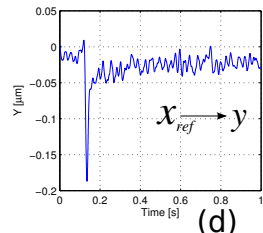

(d)
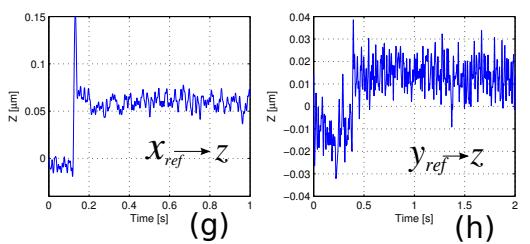

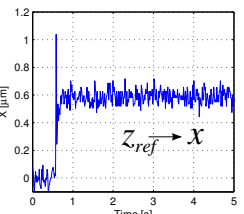

(c)

(b)

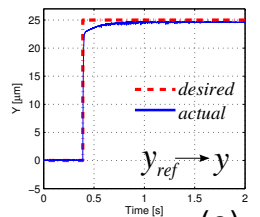

(e)

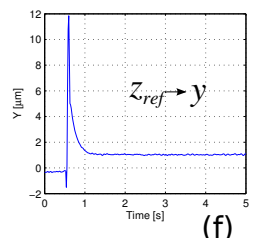

(f)

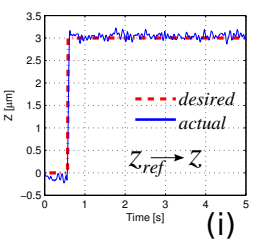

Figure 20: Verification of the transient parts for the closed-loop.

notations $A_{f}$ and $A_{m}$ (see Fig. 12-(a)) stand for the actuator deflection before the creep appears and the deflection after the time for creep evaluation (600s in our case), respectively. For the oscillations, $A_{f}$ and $A_{m}$ (see Fig. 13-(a)) stand for the maximal deflection of the step response (overshoot) and the deflection after the settling time, respectively.

Numerical values of hysteresis, creep and oscillations, for direct transfers, are represented in Tab. 1. For the open-loop system, these values are calculated based on characterization step in Section 4.2 (Fig. 11, Fig. 12 and Fig. 13). For the closed-loop system, they are calculated based on results in Fig. 18, Fig. 19 and Fig. 20.

Table 1: Numerical values of hysteresis, creep and oscillations, for direct transfers.

\begin{tabular}{cccc|ccc}
\hline \hline & \multicolumn{3}{c}{ Open-loop } & \multicolumn{3}{c}{ Closed-loop } \\
\hline & Hyst. & Creep & Oscil. & Hyst. & Creep & Oscil. \\
\hline X & $16.2 \%$ & $17.0 \%$ & $42.1 \%$ & $0.01 \%$ & $0.31 \%$ & $1.62 \%$ \\
Y & $17.1 \%$ & $7.00 \%$ & $50.6 \%$ & $0.01 \%$ & $0.04 \%$ & $0.06 \%$ \\
Z & $25.8 \%$ & $12.9 \%$ & $6.40 \%$ & $0.00 \%$ & $0.01 \%$ & $3.31 \%$ \\
\hline
\end{tabular}

As we can see, the comparison in Tab. 1 shows that hysteresis, which was more than $16.2 \%$ in direct transfers for the open-loop system, is removed $(\simeq$ $0.0 \%$ ). The amount of the creep decreases from more 
than $7.0 \%$ to less than $0.4 \%$, and the oscillations from $42.19 \%$ to less than $3.5 \%$.

To evaluate the cross-couplings, we quantify their amplitudes with respect to the corresponding direct transfers. The cross-coupling amplitude for axis $i(i \in$ $\{x, y, z\})$ is defined by the ratio $\left(H_{U_{j}}^{i} / H_{U_{i}}^{i}\right) \times 100 \%$, with $H_{U_{j}}^{i}$ the maximal deflection for the cross-coupling $U_{j} \rightarrow i(j \neq i)$, and $H_{U_{i}}^{i}$ the maximal deflection of the corresponding direct transfer $U_{i} \rightarrow i$ (the maximal deflection that we would obtain by applying $U_{i}$ ).

The cross-couplings amplitudes (Tab. 2) have been calculated based on Fig. 11 (for the open-loop system) and Fig. 18 (for the closed-loop system).

Table 2: Cross-couplings amplitudes, for open-loop and closed-loop systems.

\begin{tabular}{|c|c|c|}
\hline & Open-loop & Closed-loop \\
\hline$X$ & $\frac{H_{U_{y}}^{x}}{H_{U_{x}}^{x}}=\frac{3.4}{56.5}=6.0 \%$ & $\frac{H_{y_{r e f}}^{x}}{H_{x_{r e f}}^{x}}=\frac{0.04}{24.6}=0.1 \%$ \\
\hline axis & $\frac{H_{U_{z}}^{x}}{H_{U_{x}}^{x}}=\frac{0.7}{56.5}=1.2 \%$ & $\frac{H_{z_{r e f}}^{x}}{H_{x_{r e f}}^{x}}=\frac{0.02}{24.6}=0.0 \%$ \\
\hline $\mathrm{Y}$ & $\frac{H_{U_{x}}^{y}}{H_{U_{y}}^{y}}=\frac{1.1}{60.2}=1.8 \%$ & $\frac{H_{x_{r e f}}^{y}}{H_{y_{r e f}}^{y}}=\frac{0.02}{24.9}=0.0 \%$ \\
\hline axis & $\frac{H_{U_{z}}^{y}}{H_{U_{y}}^{y}}=\frac{1.2}{60.2}=1.9 \%$ & $\frac{H_{z_{r e f}}^{y}}{H_{y_{r e f}}^{y}}=\frac{0.01}{24.9}=0.0 \%$ \\
\hline $\mathrm{Z}$ & $\frac{H_{U_{x}}^{z}}{H_{U_{z}}^{2}}=\frac{2.4}{6.2}=38.7 \%$ & $\frac{H_{x_{r e f}}^{2}}{H_{z_{r e f}}^{2}}=\frac{0.1}{2.5}=4.0 \%$ \\
\hline axis & $\frac{H_{U_{y}}^{2}}{H_{U_{z}}^{2}}=\frac{1.2}{6.2}=19.3 \%$ & $\frac{H_{y_{r e f}}^{z}}{H_{z_{r e f}}^{2}}=\frac{0.1}{2.5}=4.0 \%$ \\
\hline
\end{tabular}

We notice that the calculated controller lowered considerably the cross-couplings amplitudes (from 6\% to less than $0.1 \%$ for $\mathrm{X}$ axis, from $1.9 \%$ to $0.0 \%$ for $\mathrm{Y}$ axis, and from $38.7 \%$ to $4.0 \%$ for $\mathrm{Z}$ axis).

\subsection{Helix trajectories tracking}

To test the ability of the closed-loop to track complex trajectories, we have forced the tip of the piezoelectric tube to track an helix shaped trajectory, by applying simultaneously a sine wave $x_{\text {ref }}$ of amplitude $15 \mu \mathrm{m}$, a cosine wave $y_{\text {ref }}$ of amplitude $15 \mu \mathrm{m}$, both at at $f=$ $0.1 \mathrm{~Hz}$, and a staircase signal $z_{\text {ref }}$ increasing from $0 \mu \mathrm{m}$ to $3 \mu \mathrm{m}$. Fig. 21 shows that the helix trajectory tracking is successfully achieved, with the tracking error less than $0.6 \mu \mathrm{m}$ for $\mathrm{X}$ and $\mathrm{Y}$ axis, and less than $0.1 \mu \mathrm{m}$ for $\mathrm{Z}$ axis.
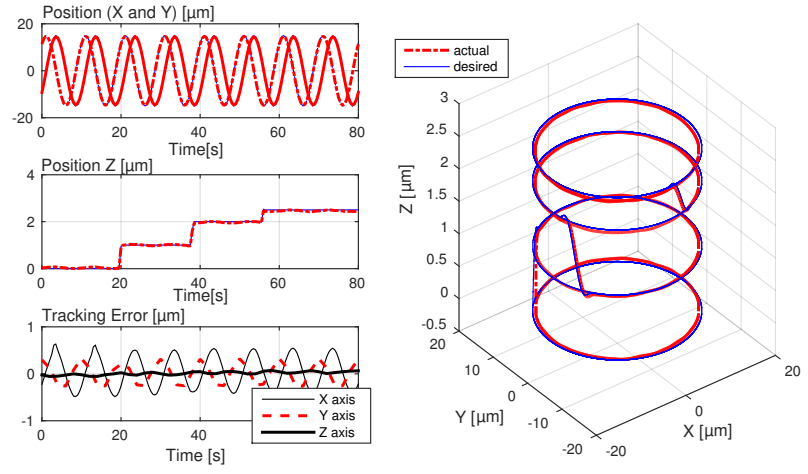

Figure 21: Helix trajectory tracking.

\section{Conclusion}

The characterization, the modeling and the $H_{\infty}$ control of $n$-DOF piezoelectric actuators has been treated in this paper, with an application to a 3-DOF nanopositioner. For that, instead of using a nonlinear and coupled multivariable system, we proposed to decouple the system and derive $n$ monovariable linear systems where the hysteresis, the creep and the cross-couplings are accounted in a direct multiplicative structured uncertainty and external fictive disturbance. The proposed technique allowed to calculate $H_{\infty}$ controller for each axis independently, with an explicit consideration of the cross-coupling effect. Additionally to the crosscouplings, to the creep and to the hysteresis nonlinearity, piezoelectric actuators exhibit badly damped oscillations which are considered as dynamics during the proposed modeling. The experimental tests carried out on a 3-DOF piezoelectric tube scanner have shown that the calculated controllers rejected the effect of the external disturbance successfully, and therefore the effect of the hysteresis, the creep and the crosscouplings. Furthermore, the results on an helix trajectory tracking confirmed the efficiency of the proposed control technique for spacial precise positioning and tracking. Nevertheless, the current research focused on modeling and control of piezoelectric systems with the same number of inputs and outputs. In future works, the proposed technique will be extended to under and over actuated systems modeling and control. Moreover, our future works will include the combination of the proposed technique with feedforward control schemes, in order to increase the overall bandwidth of the closed-loop system and to achieve the high tracking performances at very high frequencies. 


\section{References}

[1] G. Binnig, C. F. Quate, C. Gerber, "Atomic Force Microscope", Physical Review Letters, Vol. 56, pp. 930-933 (1986).

[2] G. Binnig, and D. P. E. Smith, "Single-tube three-dimensional scanner for scanning tunneling microscopy", Review of Scientific Instruments, Vol. 57, No. 8, pp. 1688-1689 (1986).

[3] D. Santosh, E. Evangelos and S. O. R. Moheimani, "A survey of Control Issues in Nanopositioning", IEEE Transactions on Control Systems Technology, Vol. 15, pp. 802-823 (2007).

[4] J. Agnus, N. Chaillet, C. Clévy, S. Dembl, M. Gauthier, Y. Haddab, G. Laurent, P. Lutz, N. Piat, K. Rabenorosoa, M. Rakotondrabe and B. Tamadazte, "Robotic Microassembly and micromanipulation at FEMTO-ST", Journal of Micro-Bio Robotics, Vol. 8, Issue 2, pp. 91-106 (2013).

[5] J. J. Dosch, D. J. Inman and E. Garcia, "A Self-Sensing Piezoelectric Actuator for Collocated Control", Journal of Intell. Mater. Syst. and Struct., Vol. 3, pp. 166-185 (1992).

[6] S. O. R. Moheimani, and Yuen K. Yong, "A new piezoelectric tube scanner for simultaneous sensing and actuation." American Control Conference, (2009).

[7] M. Rakotondrabe, I. Alexandru Ivan, S. Khadraoui, C. Clévy, P. Lutz and N. Chaillet, "Dynamic displacement self-sensing and robust control of cantilevered piezoelectric actuators dedicated to microassembly tasks", IEEE/ASME, International Conference on Intelligent Mechatronics, Montreal Canada, pp. 557-562 (2010).

[8] M. Rakotondrabe, A. Ivan, S. Khadraoui, P. Lutz and N. Chaillet, "Simultaneous displacement and force self-sensing in piezoelectric actuators and applications to robust control of the displacement", IEEE/ASME - Transactions on Mechatronics, Vol. 20, No. 2, pp. 519-531 (2015).
[9] M. Rakotondrabe, K. Rabenorosoa, J. Agnus and N. Chaillet, 'Robust Feedforward-Feedback Control of a Nonlinear and Oscillating 2-DOF Piezocantilever", IEEE T-Automation Science and Engineering, Vol. 8, pp. 506-519 (2011).

[10] M. Goldfarb, N.Celanovic, "Modeling Piezoelectric Stack Actuators for Control of Micromanipulation" , IEEE Control Systems Mag., Vol. 17, No. 3, pp. 69-79 (1997).

[11] S. Mitsuhashi, K. Wakamatsu, Y. Aihara, N. Okihara, "Relay Using Multilayer Piezoelectric Actuator", Jpn. J. of Appl. Phys., Vol. 24, No. 3, pp. 190-192 (1985).

[12] K. G. Vandervoort, et al. "Full temperature calibration from 4 to $300 \mathrm{~K}$ of the voltage response of piezoelectric tube scanner PZT-5A for use in scanning tunneling microscopes", Review of scientific instruments, Vol. 64, No. 4, pp. 896-899 (1993).

[13] M. Rakotondrabe, "Smart materials-based actuators at the micro/nano-scale: characterization, control and applications", edited book, Springer - Verlag, New York, ISBN 978-1-4614-6683-3 (2013).

[14] S. H. Chung, and E. H. K. Fung, ”Adaptive Sliding Mode Control of Piezoelectric Tube Actuator With Hysteresis, Creep and Coupling Effect", ASMEInternational Mechanical Engineering Congress and Exposition, pp. 419-428 (2010).

[15] D. Habineza, M. Rakotondrabe and Y. Le Gorrec, "Bouc-Wen Modeling and Feedforward Control of multivariable Hysteresis in Piezoelectric Systems: Application to a 3-DoF Piezotube scanner", IEEE - Transactions on Control Systems Technology, DOI.10.1109/TCST.2014.2386779.

[16] M. Rakotondrabe, C. Clévy and P. Lutz, "Complete open loop control of hysteretic, creeped and oscillating piezoelectric cantilever", IEEE - Transactions on Automation Science and Engineering, Vol. 7, No. 3, pp. 440-450 (2010). 
[17] M. Rakotondrabe, "Modeling and Compensation of Multivariable Creep in multi-DOF Piezoelectric Actuators", IEEE - International Conference on Robotics and Automation, St Paul Minnesota USA, pp. 4577-4581, (2012).

[18] D. Croft, G. Shed, and S. Devasia, "Creep, hysteresis, and vibration compensation for piezoactuators: atomic force microscopy application", Journal of Dynamic Systems, Measurement, and Control, Vol. 123, No. 1, pp. 35-43 (2001).

[19] H. Jung, J. Shim, and D. Gweon, "New openloop actuating method of piezoelectric actuators for removing hysteresis and creep", Review of Scientific Instruments, Vol. 71, pp. 3436-3440 (2000).

[20] K. K. Leang, Zou Qingze and S. Devasia, "Feedforward control of piezoactuators in atomic force microscope systems", IEEE-Control Systems, Vol. 29, No. 1, pp. 70-82 (2009).

[21] M. Rakotondrabe, "Classical Prandtl-Ishlinskii modeling and inverse multiplicative structure to compensate hysteresis in piezoactuators", American Control Conference, Montral Canada, pp. 1646-1651 (2012).

[22] M. Rakotondrabe, "Bouc-Wen modeling and inverse multiplicative structure to compensate hysteresis nonlinearity in piezoelectric actuators", IEEE - Transactions on Automation Science and Engineering, Vol. 8, Issue. 2, pp. 428-431 (2011).

[23] M. Al Janaideh, S. Rakheja, C. Y. Su , "An analytical generalized Prandtl-Ishlinskii model inversion for hysteresis compensation in micropositioning control", IEEE/ASME Transactions on Mechatronics, Vol. 16, No. 4, pp. 73-744 (2011).

[24] K. K. Tan, T. H. Lee, and H. X. Zhou, ”Micropositioning of linear-piezoelectric motors based on a learning nonlinear PID controller", IEEE/ASME Trans. Mechatron., Vol. 6, No. 4, pp. 428-436 (2001).

[25] E. Kouno, "A fast response piezoelectric actuator for servo correction of systematic errors in precision machining", Ann. CIRP, Vol. 33, No. 1, pp. 369-372 (1982).
[26] N. Tamer and M. Dahleh, "Feedback control of piezoelectric tube scanners", Proceedings of the 33rd IEEE Conference on Decision and Control, pp. 1826-1831 (1994).

[27] L. Lei, K. K. Tan, S. Chen, C. Sing Teo, and T. Heng Lee, "Discrete composite control of piezoelectric actuators for high-speed and precision scanning", IEEE Transactions Industrial Informatics, Vol. 9, No. 2, pp. 859-868 (2013).

[28] S. Salapaka, A. Sebastian, J. P. Cleveland and M. V. Salapaka, "Design, Identification and Control of a Fast Nanopositioning Device", Proceedings of the 2002 American Control Conference, Vol. 3, pp. 1966-1971 (2002).

[29] N. Chuang and I. R. Petersen, " Robust H Infinity Control of Hysteresis in a Piezoelectric Stack Actuator", IFAC World Congress, Vol. 17, No. 1, (2008).

[30] M. Rakotondrabe, Y. Haddab and P. Lutz, "Quadrilateral modelling and robust control of a nonlinear piezoelectric cantilever", IEEE Transactions on Control Systems Technology, Vol. 17, Issue. 3, pp. 528-539 (2009).

[31] M. Rakotondrabe, Y. Haddab and P. Lutz, "Plurilinear modeling and discrete -synthesis control of a hysteretic and creeped unimorph piezoelectric cantilever", IEEE - International Conference on Automation, Robotics, Control and Vision, Grand Hyatt Singapour, pp. 57-64 (2006).

[32] K. K. Leang, and D. Santosh, "Feedbacklinearized inverse feedforward for creep, hysteresis, and oscillation compensation in AFM piezoactuators", IEEE Transactions on Control Systems Technology, Vol. 15, No. 5, pp. 927-935 (2007).

[33] S. Khadraoui, M. Rakotondrabe and P. Lutz, "Design of RST-structured controller for parametric uncertain system using interval analysis: application to piezocantilever", Asian Journal of Control, Vol. 15, No. 1, pp. 142-154 (2013).

[34] S. Kuiper, and G. Schitter. "Model-based feedback controller design for dual actuated atomic force microscopy", Mechatronics, Vol. 22, No. 3, pp. 327-337 (2012). 
[35] B. J. Kenton, and K. K. Leang, "Design and Control of a Three-Axis Serial Kinematic HighBandwidth Nanopositioner", IEEE/ASME Transactions on Mechatronics, Vol. 17, No. 2, pp. 356-369 (2012).

[36] I. Al-Thani Mahmood, K. Liu, S. O. R. Moheimani, "Two Sensor Based H-Infinity Control of a Piezoelectric Tube Scanner", Proceedings of the 17th International Federation of Automatic Control World Congress, Seoul, Korea, (2008).

[37] A. Daniele, S. Salapaka, M. V. Salapaka, M. Dahleh, "Piezoelectric scanners for atomic force microscopes : design of lateral sensors, identification and control", American Control Conference, Vol. 1, pp. 253-257 (1999).

[38] C. Lee, and S. M. Salapaka, "Robust broadband nanopositioning: fundamental trade-offs, analysis, and design in a two-degree-of-freedom control framework", Nanotechnology, Vol. 20, No. 3, (2009).

[39] Y. K. Yong, L. Kexiu, and S. O. R. Moheimani, "Reducing cross-coupling in a compliant XY nanopositioner for fast and accurate raster scanning", IEEE Transactions on Control Systems Technology, Vol. 18, No. 5, pp. 1172-1179 (2007).

[40] R. Merry, M. Uyanik, R. Van De Molengraft, M. Steinbuch, R. Koops, M. Van Veghel, "Modeling and compensation of asymmetric hysteresis in a piezo actuated metrological AFM", American Control Conference, pp. 967-972 (2009).

[41] S. S. Aphale, A. Ferreira, and S. O. R. Moheimani, "A robust loop-shaping approach to fast and accurate nanopositioning", Sensors and Actuators, Vol. 204, pp. 88-96 (2013).

[42] J. L. Pons, "Emerging actuator technologies: a micromechatronic approach", ISBN 0-470-091975, Wiley, (2005).

[43] F. Preisach, "Ueber die magnetische Nachwirkung", Zeitschrift fuer Physik, Vol. 94, pp. 277302 (1935).
[44] L. Prandtl, "Ein Gedankenmodell zur kinetischen Theorie der festen Korper", Z. Angew. Math. Mech., Vol. 8, pp. 85-106 (1928).

[45] A. Y. Ishlinskii, "Some applications of statistical methods to describing deformations of bodies", Izv. Akad. Nauk SSSR, Techn. Ser., Vol. 9, pp. 580-590 (1944).

[46] R. Bouc, "Forced vibration of mechanical systems with hysteresis", Conference on Nonlinear Oscillation, Prague, (1967).

[47] Y. K. Wen, "Method for random vibration of hysteresis systems", Journal of the Engineering Mechanics Division, Vol. 102, pp. 249-263 (1976).

[48] M. Al Janaideh, Chun-Yi Su, S. Rakheja, "A generalized asymmetric play hysteresis operator for modeling hysteresis nonlinearities of smart actuators", 10th International Conference on Control, Automation, Robotics and Vision, pp. 240244 (2008).

[49] K. Zhou and J. C. Doyle, "Essentials of robust control", Prentice Hall, ISBN 0-13-525833-2, (1998).

[50] S. Skogestad, and I. Postlethwaite, "Multivariable feedback control: analysis and design", vol. 2, New York: Wiley, (2007).

[51] C. Berard, J. M. Biannic, D. Saussie, "La commande multivariable-Application au pilotage d'un avion", Dunod, (2012).

[52] K. Glover and J. C. Doyle, "State-space formulae for all stabilizing controllers that satisfy an $\mathrm{H}_{\infty}$ norm bound and relations to risk sensivity", Systems and Control Letters, Vol. 11, pp. 167-172 (1988).

[53] J. C. Doyle, K. Glover, P. K. Khargonekar and B. A. Francis, "State-space solutions to standard $\mathrm{H}_{2}$ and $\mathrm{H}_{\infty}$ control problems", IEEE Transactions on Automatic Control, Vol. 34, No. 8, pp. 831-846 (1989). 